\title{
Characterization and Modeling of Multi-Layered Diatom Biooptics for Energy- Harvesting Applications
}

\author{
Jane Yang ${ }^{*}$, Shiyou Xu ${ }^{*}$, Gerald Poirier ${ }^{*}$, Nan Yao . \\ *Princeton Institute for the Science and Technology of Materials, Princeton University, Princeton NJ
}

The purpose of this research is to provide simulation-based evidence linking diatom frustule structure and optical functionality. In creating computational models of the diatom species Isthmia nervosa that include realistic defects in the frustule pore patterns using measurements taken by scanning electron microscopy, this paper will illuminate useful information on how to create biomimetic analogues for energy-harvesting purposes.

There are over 100,000 different known species of diatoms, each with a uniquely intricate and elegantly porous silica cell wall, or frustule, composed of two overlapping halves connected by girdle bands. In the past decade, the biooptics of diatoms has come to be of interest as diatoms are photosynthetic organisms accounting for $20-25 \%$ of global primary production, or the production of chemical energy by living organisms on which all life on Earth depends. Fuhrmann et. al have suggested that diatoms can be viewed as living photonic crystal slabs due to their latticed frustules [1]. In particular, the girdle bands of a diatom may serve as waveguides. Because the chloroplasts of diatoms are located in very close proximity to the cell wall, viewing diatoms in this way may lead to advanced biologically-inspired dye-based light harvesting materials. Such materials would not only represent a significant breakthrough in photovoltaic technologies, but also optical biosensing technologies. To date, most research on diatom biooptics have focused on idealizations of a diatom frustule with pores on the nanometer scale [2]. However, diatom sizes range across several orders of magnitudes. Furthermore, diatoms have evolved natural defects that depart from perfect lattice structures. SEM and AFM images of diatoms provide enough detail to create precise models that incorporate these defects and allow for realistic simulation of diatom interactions with light.

Isthmia nervosa (pores on the $\mu \mathrm{m}$-scale; Fig. 1) samples originally from Burton, Washington were ordered from Ward's Natural Science (Product \# 52-2703) and prepared for SEM and AFM study. Lattice constants and pore diameters were measured both parallel to and perpendicular to the girdle band-valve interface of whole diatoms while thickness measurements were taken from cross-sections of broken diatoms captured on a FEI XL30 FEG-SEM. ImageJ software was used to measure and save dimensions from raw data files. RSoft's BandSOLVE software was used to calculate band structures for models based on the SEM and AFM measurements. BandSOLVE uses the wellestablished plane wave expansion (PWE) method for calculating guided modes up to the light cone within periodic slabs by solving the Helmholtz equation (Equation 1) via minimizing the Raleigh quotient (Equation 2) applied to the periodic crystal slab with an introduced third dimension of periodicity. The PWE assumes a periodic layering of slabs separated by enough background region such that the slabs do not interfere with evanescent waves from neighboring slabs.

$$
\hat{\mathbf{L}} \mathbf{u}_{\mathbf{k}}=(i \mathbf{k}+\nabla) \times\left(\frac{1}{\epsilon(\mathbf{x})}(i \mathbf{k}+\nabla)\right) \times \mathbf{u}_{\mathbf{k}}=\left(\frac{\omega}{c}\right)^{2} \mathbf{u}_{\mathbf{k}}
$$




$$
E\left[\mathbf{u}_{\mathbf{k}}(\mathbf{x})\right]=\frac{\int d \mathbf{x}^{3} \mathbf{u}_{\mathbf{k}}^{*}\left[\hat{\mathbf{L}} \mathbf{u}_{\mathbf{k}}\right]}{\int d \mathbf{x}^{3} \mathbf{u}_{\mathbf{k}}^{*} \mathbf{u}_{\mathbf{k}}}
$$

SEM image analysis showed that Isthmia nervosa girdle band pores are slightly elongate in shape $\left(r_{I}\right.$ $\left.=3.59 \pm 0.47 \mu \mathrm{m}, r_{2}=4.03 \pm 0.80 \mu \mathrm{m}\right)$. Furthermore, their periodicity differs depending on the axis of measurement $\left(a_{1}=6.58 \pm 0.54 \mu \mathrm{m}, a_{2}=7.58 \pm 1.31 \mu \mathrm{m}\right)$. A linear waveguide composed of larger pores $\left(r_{3}=6.76 \pm 1 \mu \mathrm{m}, r_{4}=5.98 \pm 1.61 \mu \mathrm{m}\right)$ also manifests at the girdle band-valve interface. Most interestingly, a porous thin film (with thickness $h_{\text {thin }}=0.84 \pm 0.16 \mu \mathrm{m}$ compared with the frustule thickness $h_{\text {frustule }}=2.38 \pm 1.16 \mu \mathrm{m}$ ) covers the $\mu \mathrm{m}$-scale pores. ImageJ analysis indicates the thin film is approximately $15 \%$ porous. A full-scale simulation of the Isthmia nervosa girdle band in air with the domain shown in Fig. 2 indicated that the diatom species was designed to increase light/matter

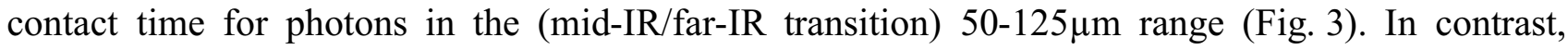
Yamanaka et. al suggested a preference for blue light interaction [2]. These results assume the thin film refractive index is identical to that of the overall frustule $(\eta=1.46)$, though $\mathrm{x}$-ray diffraction results from Desikachary [3] suggest the presence of iron in Isthmia nervosa, which may alter the refractive index and thus the band structure. In addition to refraction index, initial sensitivity analyses suggest the lattice constant and frustule thicknesses are the most significant parameters that affect optical performance.

This paper will demonstrate the optical effect of a cascade pore pattern due to the porous thin film in a $\mu \mathrm{m}$-scale diatom and will discuss the influence of various design parameters on optical efficiency for application purposes [4].

\section{References}

[1] T. Fuhrmann, S. Landwehr, M. El Rharbi-Kucki, and M. Sumper, Applied Physics B. 78(2004) 257-260.

[2] S. Yamanaka, R. Yano, H. Usami, N. Hayashida, M. Ohguchi, H. Takeda, and K. Yoshino, Journal of Applied Physics. 103(2008) 1-5.

[3] T.V. Desikachary and N.E. Dweltz, Proc. Indian Acad. Sci. 53(1961) 157-165.

[4] This research is supported by the Princeton University Lidow Senior Thesis Fund.

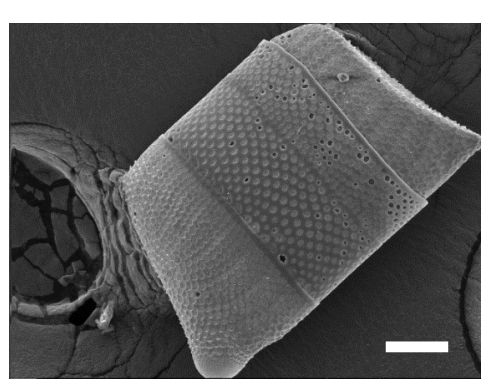

Figure 1.

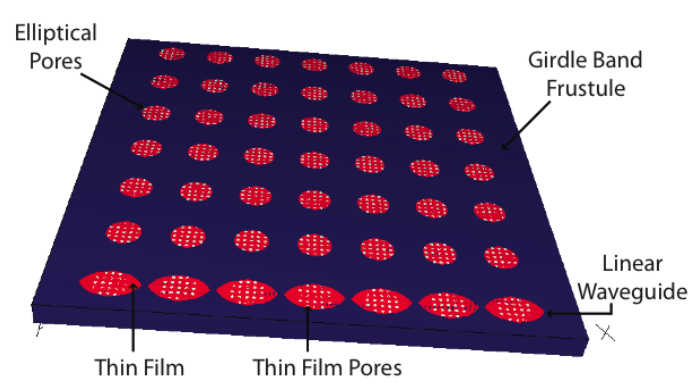

Figure 2.

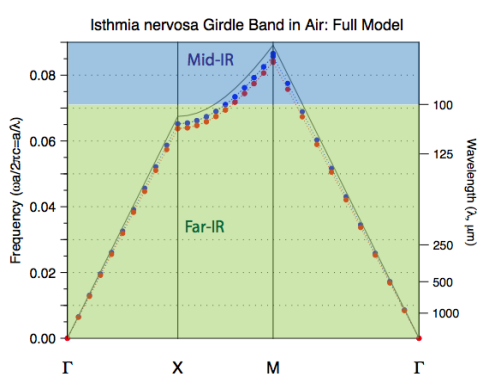

Figure 3.

FIG. 1. SEM image of an Isthmia nervosa diatom showing both the valves and girdle band. The scale bar represents $50 \mu \mathrm{m}$.

FIG. 2. 3D rendering of girdle band model (created with RSoft BandSOLVE) including a detailed porous thin film covering the large diatom pores.

FIG. 3. Band diagram for the girdle band model shown in FIG. 2, as solved by RSoft BandSOLVE. 